Immer mehr junge Zahnärztinnen und Zahnärzte scheuen das Investitionsrisiko einer eigenen Niederlassung und ziehen daher das Angestelltenverhältnis als Beschäftigungsmöglichkeit vor.

In anderen freien Berufen, wie bei Rechtsanwälten und Steuerberatern, ist es üblich, mit angestellten Berufsträgern zu arbeiten, ohne dass es die Existenz dieser freie Berufe je in Frage gestellt hätte.

Es gilt also, die Chancen zu sehen, die ein Z-MVZ den Zahnärzten bieten kann. Da bisher die Anstellung von Zahnärzten nur sehr eingeschränkt möglich war, haben viele Praxen in der Vergangenheit weitere Behandler über rechtlich bedenkliche Partnerschaften integriert. Sogenannte Juniorpartnerverträge, mit denen man Gesellschafter ohne Kapitalbeteiligung in die Praxis aufgenommen hat. Solche Konstruktionen sind üblich, aber leider in allzu vielen Fällen nicht an die aktuelle Rechtsprechung zu diesem Thema angepasst worden. Seit einem Urteil des Bundessozialgerichts aus dem Jahr 2010 und einem Urteil des Finanzgerichts Düsseldorf aus dem Jahr 2013 beinhalten solche Verträge oftmals erhebliche Regressgefahren und Probleme mit der Gewerbesteuer. Diese Probleme werden den niederge- lassenen Zahnärzten meist erst bewusst, wenn es zu spät ist. Was viele auch nicht wissen: Wenn sie sich mit einem Partner zu einer Gemeinschaftspraxis zusammenschließen, kann man sich des Partners, wenn sich die Zusammenarbeit doch nicht als so gedeihlich wie geplant erweist, nicht einfach durch Kündigung entledigen, da es im deutschen Gesellschaftsrecht kein „Hinauskündigunsgrecht" für unliebsame Partner gibt. Einer der Gründe, warum viele Trennungen von Praxispartnern sehr unerfreulich und mit erheblichen finanziellen Konsequenzen für die Beteiligten Partner verlaufen.

Was das Z-MVZ also leisten kann, ist den beteiligten Zahnärzten eine Struktur zur Verfügung zu stellen, die es nicht mehr erforderlich macht, gezwungenermaßen Partner oder Juniorpartner aufzunehmen.

\section{MVZ als zu präferierende Versorgungsform?}

Natürlich nicht. Wie immer bedarf es einer Einzelfallabwägung, welche Versorgungsform für die konkrete Praxis die richtige ist. Pauschale Antworten sind völlig fehl am Platz. Die Einzelpraxis

\title{
Zwischenruf von Dr. K. Ulrich Rubehn: In einem Boot
}

Die Hauptversammlung in Bonn hat im vergangenen Oktober das Thema „Angestellte Zahnärzte im Freien Verband “ in der richtigen Richtung diskutiert: Die Zahnärzteschaft braucht eine geschlossene Vertretung ihrer Interessen gegenüber der Politik. Dort, wo den Kammern und Kassenzahnärztlichen Vereinigungen (KZVen) aufgrund ihres Körperschaftsstatus die Hände gebunden sind, ist das Feld des Freien Verbandes Deutscher Zahnärzte (FVDZ). Der FVDZ ist zugleich notwendig, um den Zahnärztekammern und KZVen den Willen der Zahnärzteschaft zu vermitteln. Kooperation und gemeinsames Handeln als Zahnärzteschaft gegenüber der Politik ist dabei Voraussetzung für jegliche Chance, Gehör zu finden.

Angestellte Zahnärztinnen und Zahnärzte sind ein wichtiger Teil des Berufsstands. Die Gesetzgebung hat dem Trend zu mehr angestellten Zahnärzten Raum gegeben, der auch zunehmend von den niedergelassenen Zahnärzten genutzt wird. Viele, insbesondere Zahnärztinnen, starten als Angestellte in den Beruf. Das hat ganz unterschiedliche Gründe. Höher gewordene Hürden für die Finanzierung einer eigenen Praxis oder reduzierte Gewinnaussichten spielen dabei ebenso eine Rolle wie nicht abgeschlossene Lebensplanungen oder die Absicht, nur in Teilzeit arbeiten zu wollen.

\section{Angestellter Zahnarzt = Freiberufler?}

Für viele stellt sich die Frage, inwieweit ein angestellter Zahnarzt überhaupt Freiberufler sein kann. Natürlich eröffnet die zahnärztliche Tätigkeit in Selbstständigkeit die größten Freiräume zur Ausübung des Berufs in Selbstbestimmung. Nirgendwo sind die Erfolgschancen aber auch die Risiken größer. Entscheidendes Merkmal der freiberuflichen Tätigkeit ist aber nicht die vollständige wirtschaftliche Unabhängigkeit, vielmehr ist für das freiberufliche Handeln die Unabhängigkeit im Kernbereich des Zahnarztberufs maßgebend: Und das sind Diagnose, Beratung und Therapie. Die Kernkompetenz des Zahnarztes ist nicht die wirtschaftliche Praxisführung sondern sein (zahn-)ärztliches Handeln. Anders gesagt: Wir sind als selbstständige Zahnärzte zwar auch Kaufmann/ Kauffrau, aber in erster Linie Heilberufler. Unsere Beratung und Therapieempfehlung richtet sich dementsprechend nicht vordringlich nach wirtschaftlichen Interessen, sondern allein danach, was dem Patienteninteresse dient.

Auch formal gehören die angestellten Zahnärzte zu uns Freiberuflern. Sie sind Pflichtmitglieder der Zahnärztekammer, sie sichern ihre Altersversorgung beim berufsständischen Versorgungswerk ab und ihre Tätigkeit unterliegt - sofern in einer „Kassenpraxis" für gesetzlich Versicherte erbracht - den Regeln der KZV.

In beruflich-fachlicher Hinsicht, nämlich den zahnärztlichen Fort- und Weiterbildungen, liegen die Interessen beider Gruppen auf einer Ebene. Die politisch motivierten Regelwerke für die Zahnarztpraxen treffen den angestellten Zahnarzt mittelbar genauso wie den Praxisinhaber.

\section{Gemeinsame Interessen}

Der angestellte Zahnarzt hat in gleichem Maß ein Interesse an einer unabhängigen Berufsausübung wie sein „Chef“. Viele der Angestellten planen die spätere Niederlassung in eigener Praxis oder in einer Praxiskooperation. Sie sind in ihrem Angestelltenstatus quasi auf dem Weg in die Selbstständigkeit. Die niedergelassenen Zahnärzte haben zweifelsohne ein hohes Interesse an dieser Entwicklung - hängt doch auch die Frage der späteren Veräußerung ihrer Praxis daran. Die politischen Rahmenbedingungen können für die Zahnärzte nur dann attraktiv bleiben, wenn wir als Niedergelassene unsere Interessen gebündelt vertreten. Nur so lässt sich der Weg auf die Schlachtbank krankenkassengeführter Versorgungszentren verhindern. Die dortigen Interessen liegen klar auf dem Tisch: Kosten sparen, den Mangel verwalten und stille Rationierungen einführen. Deren Ziel ist jedenfalls nicht, den Interessen der Patienten zu dienen. Es geht wie immer um Macht.

Angestellte und niedergelassene Zahnärzte und Zahnärztinnen sitzen also in einem Boot. Und das Boot heißt: Erhalt der freien Berufsausübung. Nur ein wirtschaftlich unabhängiger und von Bevormundung freier Zahnarzt kann den Interessen seiner Patienten optimal gerecht werden.

An einem Punkt jedoch könnten die Interessen auseinanderdriften, und zwar hinsichtlich der Entlohnung des angestellten Zahnarztes und in puncto Gestaltung des Anstellungsvertrags.

Der Freie Verband kann keine gewerkschaftliche Interessenvertretung angestellter Zahnärzte übernehmen. Aber er kann sich eines zur Aufgabe machen: Modelle zur Entlohnung zu entwickeln und Empfehlungen zu geben, die gegebenenfalls dem selbstständigen Freiverbändler als Richtschnur dienen. Dumpinggehälter oder nicht leistungsgerechte Bezahlung gehören nicht zum Programm des FVDZ. Im Gegenteil: Wer seinen Berufsnachwuchs im Boot haben möchte, der sorge bitte auch dafür, dass der sich im Freien Verband ebenfalls vertreten fühlt. Berufsethik macht sich auch an diesem Punkt fest.

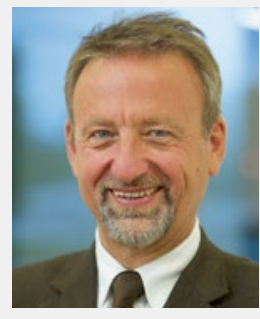

Dr. K. Ulrich Rubehn

war FVDZ-Bundesvorstandsmitglied von 2001 bis 2005, danach vier Jahre stellvertretender Bundesvorsitzender und 2009/2013 Präsident der Zahnärztekammer SchleswigHolstein. 